|| ISSN(online): 2589-8698 || ISSN(print): 2589-868X ||

International Journal of Medical and Biomedical Studies

Available Online at www.ijmbs.info

PubMed (National Library of Medicine ID: 101738825)

Index Copernicus Value 2018: 75.71

Original Research Article

Volume 3, Issue 10; October: 2019; Page No. 327-333

PROBIOTIC ADMINISTRATION IN PERIODONTAL THERAPY - A RANDOMIZED CONTROLLED CLINICAL TRIAL

Kumari Upasana ${ }^{1}$, Shivendra Chaudhary ${ }^{2}$, Pratibha P Kundapur ${ }^{3}$, Shobha Kamath ${ }^{4}$, Pentapati K Chakravarthy ${ }^{5}$, Shilpa Srinivas Kamenahalli ${ }^{6}$, Vikas Kumar ${ }^{7}$

${ }^{1}$ Assistant Professor, Department of Dentistry, Sri Krishna Medical College and Hospital, Muzaffarpur, Bihar, India

${ }^{2}$ Associate Professor and HOD, Department of Dentistry, Patna Medical College, Patna, Bihar, India

${ }^{3}$ Professor, Department of Periodontics, Manipal College of Dental Sciences, Manipal, Manipal University, India

${ }^{4}$ Professor, Department of Biochemistry, Kasturba Medical College, Manipal, Manipal University, India

${ }^{5}$ Associate Professor, Department of Public Health Dentistry, Manipal College of Dental Sciences, Manipal, Manipal University, India

${ }^{6}$ Tutor, Department of Biochemistry, Sapthagiri Institute of Medical Sciences \& Research Centre

No-15, Chikkasandra, Hesaraghatta Main Road, Bangalore, India

7 Tutor, Department of Biochemistry, Narayan Medical College, Jamuhar, Sasaram, Bihar, India.

Article Info: Received 06 October 2019; Accepted 28 October 2019

DOI: https://doi.org/10.32553/ijmbs.v3i10.761

Corresponding author: Shivendra Chaudhary

Conflict of interest: No conflict of interest.

Abstract:

Background: The mouth, like the rest of the gastrointestinal tract, is heavily colonized by bacteria. The gingival crevice between the teeth and the gums / gingivae offers a unique niche for colonization of bacteria. Gum disease or 'periodontitis' is largely attributed to a microbial etiology along with recent observations on the role of the host response. Hence, periodontal therapy has traditionally focused on the reduction of the bacterial threat. This, however, disrupts the microbial ecological balance. Probiotics is a newer treatment approach that focuses on restoring the reduced numbers of beneficial bacteria.

Methods: The present study intended to evaluate the effect of probiotic capsule administration in 20 patients each with gingivitis and periodontitis. 'Cases' received probiotic capsule and oral prophylaxis while the remaining ten patients received only oral prophylaxis and served as 'Controls' in each of the gingivitis / periodontitis groups. Plaque, Bleeding and Gingival Indices, Pocket probing depth along with GCF samples for estimation of Myeloperoxide and Thiol antioxidants were recorded at baseline and one month after treatment.

Results: Improvement of clinical parameters as well as increase in GCF thiol and decreased myeloperoxidase concentrations was observed in the groups with probiotics.

Discussion: A greater reduction in plaque scores in the cases may be attributed to probiotic induced reduced microbial colonization. Probiotic species effectively reduce the level of inflammation associated molecules, such as prostaglandin E2 and interferon $\mathrm{C}$ and MMP activity. It may be plausible that by modulating these factors, probiotics can lead to improvement of the periodontal parameters.

Conclusion: Hence, probiotic supplementation along with scaling and root planing may be an effective adjunct for periodontal therapy.

Keywords: Antioxidants, capsule, gingival crevicular fluid, gingivitis, periodontitis, probiotics

\section{1 Introduction:}

The oral cavity is an integral part of the gastro intestinal tract along with the esophagus, the stomach, the small and large intestine. Like the rest of the gastrointestinal tract, the oral cavity is heavily colonized by bacteria. The gingival crevice between the teeth and the gums / gingivae offers a unique niche for colonization of bacteria.

Gum diseases or 'periodontal' diseases like gingivitis and periodontitis are a consequence of changes in the ecology along with complex interactions between the pathogenic bacteria of the biofilm and the host's inflammatory immune response. Since it is difficult to influence the host response, traditional periodontal 
therapies focused on the reduction of the bacterial threat. ${ }^{1}$ This shifts the sub gingival microbiota to a less pathogenic composition and it also reduces overall microbial load. ${ }^{2}$ Newer treatment approaches have started to focus on a possible third etiological factor, 'the reduction or absence of the so called beneficial bacteria' and restoring these reduced numbers of beneficial bacteria. In this regard probiotics might be of considerable interest in the prevention and treatment of microbial plaque related periodontal diseases.

The present study aims to assess the influence of probiotic capsule supplementation on patients with gingivitis and periodontitis by evaluating clinical and biochemical parameters.

\subsection{Materials and Method:}

The present randomized clinical trial was initiated only after approval from the Institutional Ethics Committee. 40 patients, in the age range of 18-50 years, were included in the present study and were grouped as having 'Gingivitis' and 'Periodontitis.' Patients with 'Gingivitis' were defined as those with a Gingival Index $\geq 1$ and probing depth $\leq 3 \mathrm{~mm}$. 'Periodontitis' was considered when probing depth of $\geq 5 \mathrm{~mm}$ and a clinical attachment loss of $\geq 3 \mathrm{~mm}$ was observed clinically. Patients in either group were further divided into cases and controls, based on treatment protocol provided. Ten of the 20 patients with gingivitis / periodontitis received oral prophylaxis and probiotic capsule administration and were considered as 'Cases'. The remaining ten patients in the group received only oral prophylaxis and served as 'Controls'.

Patients who reported with systemic involvement and those on chemotherapeutics were excluded from the study. Tobacco use, history of periodontal treatment in last 3 months, orthodontic and prosthetic appliances were also factors for exclusion from the study.

Following initial screening, clinical measurements of Plaque Index (Silness and Loe, 1964), Bleeding index (Modified Sulcular Bleeding Index, Mombelli et al, 1987), Gingival Index (Loe and Silness, 1963), Pocket probing depth (PPD), measured from the gingival margin to the base of the pocket, and Clinical attachment loss (CAL), measured from the CementoEnamel junction to the base of the pocket were recorded. The measurements were made with a William's periodontal probe. Further, gingival crevicular fluid samples were also collected from these patients for biochemical estimation of Myeloperoxide, an oxidative stress indicator and Thiol antioxidant concentration at baseline and one month interval after treatment. Cases were advised to take one probiotic capsule (PrePro ${ }^{\circledR}$ HS, Fourrts India Laboratories Pvt Ltd.) twice daily after meals. They were also asked to immediately report any adverse effects associated with probiotic supplementation on their general health.

Data were analyzed using SPSS 14 version. Paired ttest was used when comparisons were made at baseline and follow up for each group. Independent samples t-test was used when comparing the mean change in parameters for the groups from the baseline to follow-up between cases and controls. A $p$-value of $<0.05$ was considered to be significant.

\subsection{Results:}

The mean age for gingivitis cases and controls was $24.40 \pm 3.75$ and $27.60 \pm 6.67$ years respectively. The periodontitis cases and controls had a mean age of $44.60 \pm 8.49$ and $43.50 \pm 11.67$ years respectively, which was not significantly different. There was no significant difference observed for gender as well among the cases and controls (Table A.1)

\subsubsection{Gingivitis group:}

\subsubsection{Changes in clinical parameters after intervention}

Both the case and control groups showed a statistically significant improvement in clinical parameters after one month. The mean value for $\mathrm{PI}$, $\mathrm{GI}$, and BOP decreased to $0.67 \pm 0.26,0.66 \pm 0.28$ and $0.62 \pm 0.29$ in cases and $0.80 \pm 0.24,0.80 \pm 0.22$ and $0.78 \pm 0.25$ in controls respectively (Table B.1; Graph 1). Comparison between cases and controls showed significantly greater improvement in $\mathrm{PI}(\mathrm{p}=0.04)$ and $\mathrm{GI}(p=0.023)$ in the case group as compared to the control group (Table B.2).

\subsubsection{Changes in MPO Levels}

After 4-weeks of intervention, the MPO levels decreased significantly in both the groups ( $p$ value $<0.001$ ) (Table B.1; Graph 4).The percentage reduction in the test group was more $(31.7 \pm 10.67)$ as compared to controls $(29.78 \pm 8.74)$ but the difference between the groups was not statistically significant $(p=0.662)$ (Table B.2).

\subsubsection{Changes in Total Thiol Levels}


The mean value of total thiol in cases and controls was $28.30 \pm 3.34 \mu \mathrm{mol} / \mathrm{l}$ and 29.06 $\pm 3.59 \mu \mathrm{mol} / \mathrm{l}$ respectively which was almost comparable at baseline. After intervention the thiol levels of both the groups increased significantly $(p<0.001)$ reaching a mean value of $46.65 \pm 6.36 \mu \mathrm{mol} / \mathrm{l}$ in cases and 42.22 $\pm 5.87 \mu \mathrm{mol} / /$ in controls (Table B.1; Graph 5). When comparison was done at follow up in the gingivitis group, more improvement of thiol was noted in the cases $(64.5 \% \pm 5.72)$ as compared to controls $(45.78 \% \pm 15.42)$, which was statistically significant $(p<0.004)$ (Table B.2).

\subsubsection{Periodontitis Group:}

\subsubsection{Clinical Parameters}

The baseline values of $\mathrm{PI}, \mathrm{GI}, \mathrm{BI}$ were $1.65 \pm 0.41$, $1.58 \pm 0.39$ and $1.60 \pm 0.34$ for the cases and $1.66 \pm 0.3$, $1.42 \pm 0.30$ and $1.60 \pm 0.30$ for controls respectively. After intervention, there was statistically significant improvement in clinical parameters in both cases and controls $(p<0.001)$ reaching a mean value of $0.76 \pm 0.29,0.78 \pm 0.33$ and $0.76 \pm 0.29$ in cases and $1.03 \pm 0.27,0.86 \pm 0.17,0.97 \pm 0.33$ in controls for $\mathrm{PI}, \mathrm{Gl}$, and BOP respectively (Table C.1; Graph 2). When the intergroup comparison was done, there was significantly more improvement in clinical parameters in the group supplemented with Probiotics (Graph 2).

The mean PPD and CAL for cases and controls were $4.51 \pm 0.27,1.64 \pm 0.26$ and $4.40 \pm 0.17,1.61 \pm .20$ respectively. Following non-surgical therapy the mean PPD \& CAL reduced to $3.33 \pm 0.33 \& 0.68 \pm 0.32$ in cases and $3.39 \pm 0.27$ and $0.86 \pm 0.12$ in controls. (Table C.1; Graph 3).When the cases and controls were compared at follow up, there was a statistically significant reduction in PPD of $26.28 \% \pm 5.59$ in cases and only $21.13 \% \pm 4.54$ in controls $(p=0.044)$ (Table C.2). Statistically significant difference was also observed in the CAL gain seen in both the groups, which was greater in cases as compared to controls $(p=0.013)$ (Table C.2).

\subsubsection{Changes in MPO}

Decrease in MPO levels was observed intra group in both cases and controls (Table C.1, Graph 4). Following intervention MPO levels decreased to $35.33 \pm 6.5 \%$ in cases, which was significantly greater $(p=0.013)$ as compared to controls $(25.84 \pm 8.81 \%)$ (Table C.2).

\subsubsection{Changes in THIOL levels}

There was a statistically significant increase in thiol levels after intervention in both the groups. The levels increased to $35.19 \pm 7.14$ from baseline levels of $21.97 \pm 3.43$ in cases and $37.58 \pm 7$ from baseline levels of 25.16 \pm 3.98 in controls (Table C.1, Graph 5). When compared between the groups the percentage increase of thiols was $59.05 \pm 9.61$ in cases as compared to $47.74 \pm 11.31$ in controls and this difference was statistically significant $(p=0.027)$ (Table C.2).

Patients were also asked about their general health during the period of probiotic supplementation. No unpleasant experiences related to the ingestion of these supplements were reported. In fact, they noticed improvement in their gastro intestinal and general health.

Table A.1: Gender distribution of subjects

Group

Gingivitis

[Cases]

Gingivitis [Controls]

Periodontitis [Cases]

Periodontitis [Controls]

Chi square test

\section{Gender}

Male [N] Female [N]

4

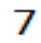

9

7 $p$ value

6

0.185 [NS]

3

1

0.291 [NS] 
Table B.1: Comparison of mean changes in parameters (clinical and biochemical) at baseline and follow up in gingivitis patients

\begin{tabular}{|c|c|c|c|c|c|c|c|c|}
\hline & \multirow[t]{2}{*}{ Parameters } & \multicolumn{2}{|c|}{ Baseline } & \multicolumn{2}{|c|}{ Follow-up } & \multicolumn{2}{|c|}{ Difference } & \multirow{2}{*}{$\begin{array}{l}\text { Pvalue } \leq 0.05 \\
\text { Significant }\end{array}$} \\
\hline & & Mean & SD & Mean & SD & Mean & SD & \\
\hline \multirow[t]{5}{*}{ GINGIVITIS (CASES) } & $\mathrm{Pl}$ & 1.54 & 0.37 & 0.67 & 0.26 & 0.87 & 0.22 & $<0.001$ \\
\hline & $\mathrm{Bl}$ & 1.52 & 0.37 & 0.62 & 0.29 & 0.90 & 0.30 & $<0.001$ \\
\hline & Gl & 1.43 & 0.35 & 0.66 & 0.28 & 0.76 & 0.20 & $<0.001$ \\
\hline & MPO & 0.16 & 0.01 & 0.11 & 0.02 & 0.05 & 0.02 & $<0.001$ \\
\hline & THIOL & 28.30 & 3.34 & 46.65 & 6.36 & -18.35 & 3.18 & $<0.001$ \\
\hline \multirow[t]{5}{*}{ GINGIVITIS (CONTROLS) } & $\mathrm{Pl}$ & 1.36 & 0.12 & 0.80 & 0.24 & 0.56 & 0.29 & $<0.001$ \\
\hline & $\mathrm{Bl}$ & 1.45 & 0.20 & 0.78 & 0.25 & 0.66 & 0.17 & $<0.001$ \\
\hline & Gl & 1.30 & 0.29 & 0.80 & 0.22 & 0.50 & 0.29 & $<0.001$ \\
\hline & MPO & .17 & 0.01 & 0.12 & 0.02 & 0.05 & 0.01 & $<0.001$ \\
\hline & THIOL & 29.06 & 3.59 & 42.22 & 5.87 & -13.16 & 4.40 & $<0.001$ \\
\hline
\end{tabular}

Table C.1: Comparison of mean changes in parameters (clinical and biochemical) at baseline and follow up in periodontitis patients

\begin{tabular}{|c|c|c|c|c|c|c|c|c|}
\hline & \multirow[t]{2}{*}{ Parameters } & \multicolumn{2}{|c|}{ Baseline } & \multicolumn{2}{|c|}{ Follow-up } & \multicolumn{2}{|c|}{ Difference } & \multirow{2}{*}{$\begin{array}{l}P \text { value } \leq 0.05 \\
\text { Significant }\end{array}$} \\
\hline & & Mean & SD & Mean & SD & Mean & SD & \\
\hline \multirow[t]{7}{*}{ PERIODONTITIS (CASES) } & $\mathrm{PI}$ & 1.65 & 0.41 & 0.76 & 0.29 & 0.87 & 0.22 & $<0.001$ \\
\hline & $\mathrm{BI}$ & 1.6 & 0.34 & 0.76 & 0.29 & 0.85 & 0.17 & $<0.001$ \\
\hline & GI & 1.58 & 0.39 & 0.78 & 0.33 & 0.8 & 0.13 & $<0.001$ \\
\hline & PPD & 4.51 & 0.27 & 3.33 & 0.33 & 1.19 & 0.28 & $<0.001$ \\
\hline & CAL & 1.64 & 0.26 & 0.68 & 0.32 & 0.97 & 0.17 & $<0.001$ \\
\hline & MPO & 0.2 & 0.04 & 0.13 & 0.02 & 0.07 & 0.02 & $<0.001$ \\
\hline & THIOL & 21.97 & 3.43 & 35.19 & 7.14 & -3.22 & 3.77 & $<0.001$ \\
\hline \multirow[t]{7}{*}{ PERIODONTITIS (CONTROLS) } & $\mathrm{PI}$ & 1.66 & 0.36 & 1.03 & 0.27 & 0.64 & 0.21 & $<0.001$ \\
\hline & $\mathrm{BI}$ & 1.6 & 0.3 & 0.97 & 0.33 & 0.63 & 0.22 & $<0.001$ \\
\hline & $\mathrm{GI}$ & 1.42 & 0.21 & 0.86 & 0.17 & 0.56 & 0.22 & $<0.001$ \\
\hline & PPD & 4.4 & 0.17 & 3.39 & 0.27 & 1.02 & 0.34 & $<0.001$ \\
\hline & CAL & 1.61 & 0.2 & 0.86 & 0.12 & 0.75 & 0.19 & $<0.001$ \\
\hline & MPO & 0.21 & 0.04 & 0.15 & 0.03 & 0.05 & 0.03 & $<0.001$ \\
\hline & THIOL & 25.16 & 3.98 & 37.58 & 7.00 & -2.41 & 4.14 & $<0.001$ \\
\hline
\end{tabular}

(Paired $\mathrm{t}$ test) 
Table B.2: Comparison of changes in clinical and biochemical parameters at follow up in gingivitis patient (expressed in percentage)

\begin{tabular}{lllllll} 
& Parameters & Cases & \multicolumn{3}{c}{ Controls } & P value $\leq 0.05$ \\
GINGIVITIS & Mean & SD & Mean & SD & Significant \\
& PI & 56.99 & 11.78 & 40.60 & 20.28 & $\mathbf{0 . 0 4}$ \\
& BI & 59.65 & 17.22 & 46.63 & 14.04 & 0.08 \\
GI & 54.53 & 12.33 & 37.16 & 18.34 & $\mathbf{0 . 0 2 3}$ \\
& MPO & 31.71 & 10.67 & 29.78 & 8.74 & 0.662 \\
& THIOL & -64.50 & 5.72 & -45.78 & 15.42 & $\mathbf{0 . 0 0 4}$
\end{tabular}

Independent sample $t$ test

Table C.2: Comparison of changes in clinical and biochemical parameters at follow up in periodontitis patients (expressed in percentage)

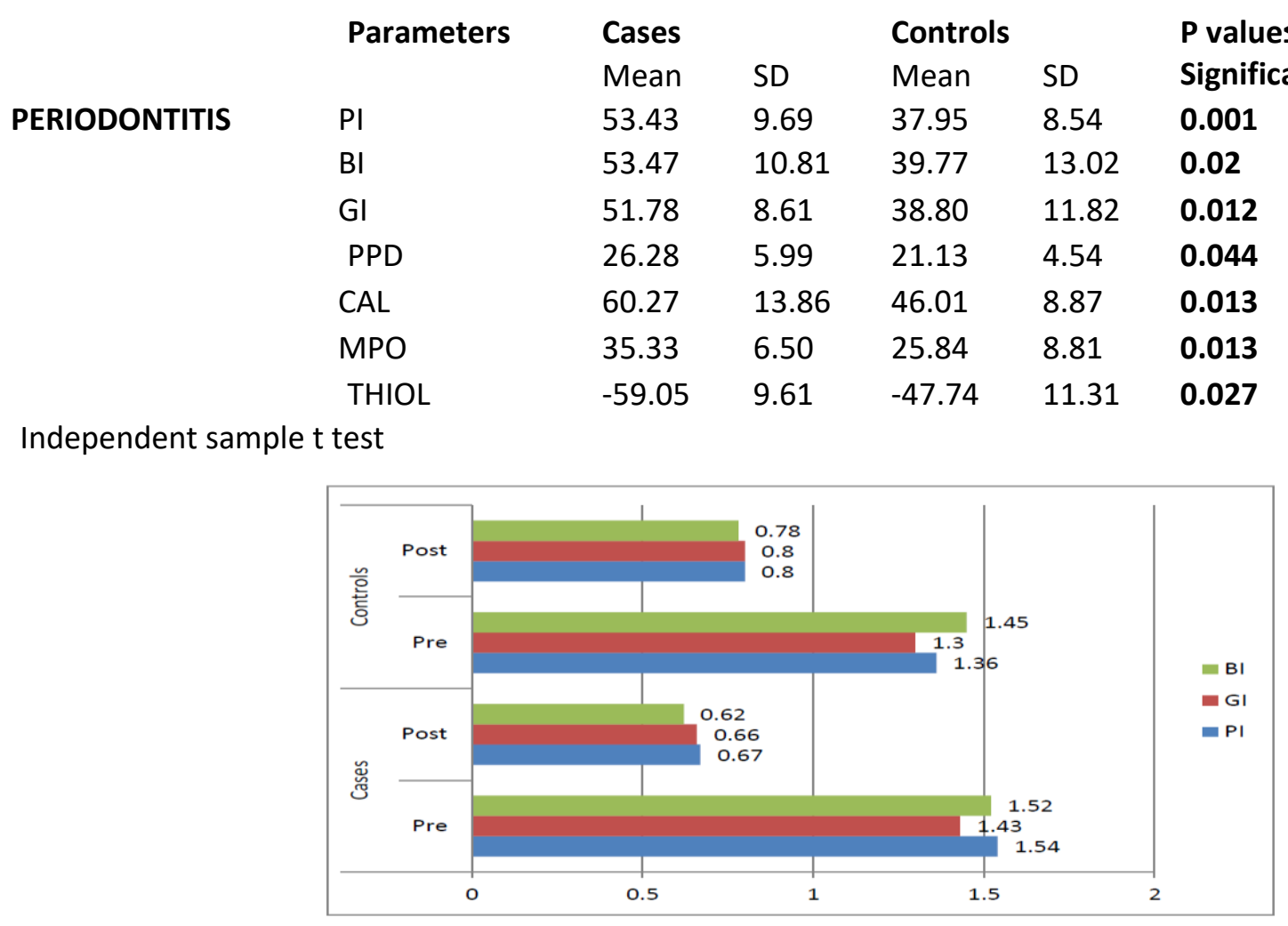

Graph 1: COMPARISON OF CLINICAL PARAMETERS IN GINGIVITIS PATIENTS

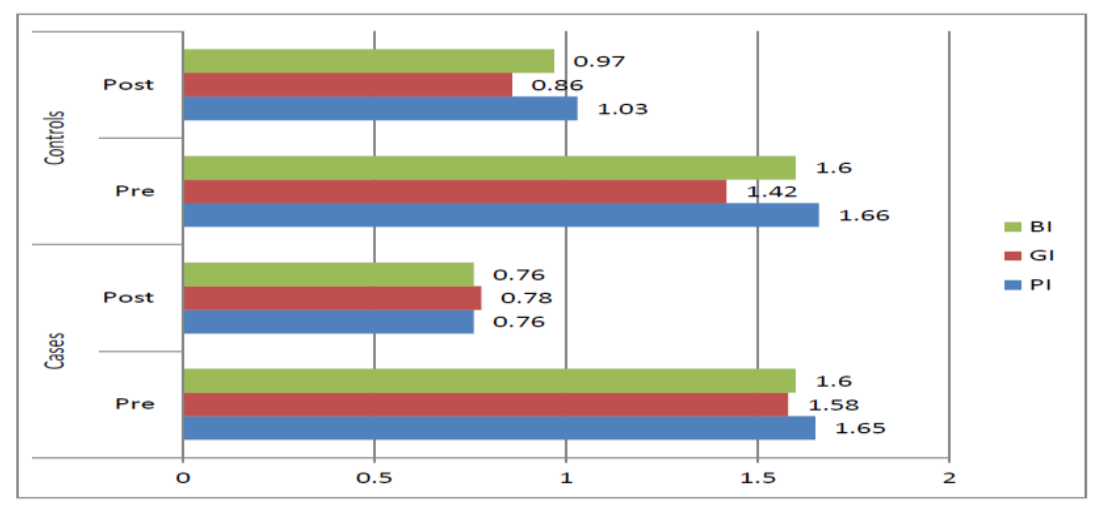

Graph 2: COMPARISON OF CLINICAL PARAMETERS IN PERIODONTITIS PATIENTS 


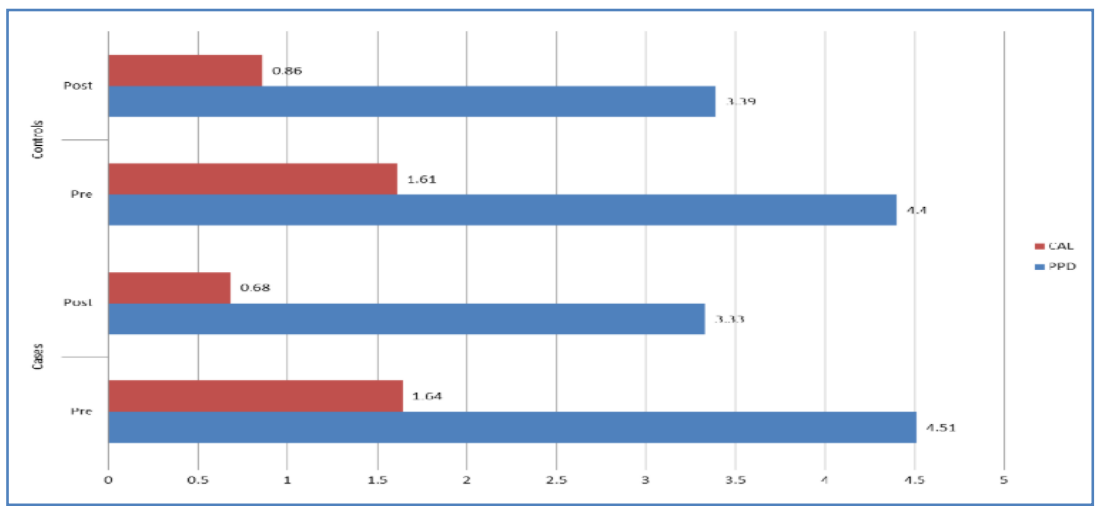

Graph 3: COMPARISON OF PPD \& CAL IN PERIODONTITIS PATIENTS

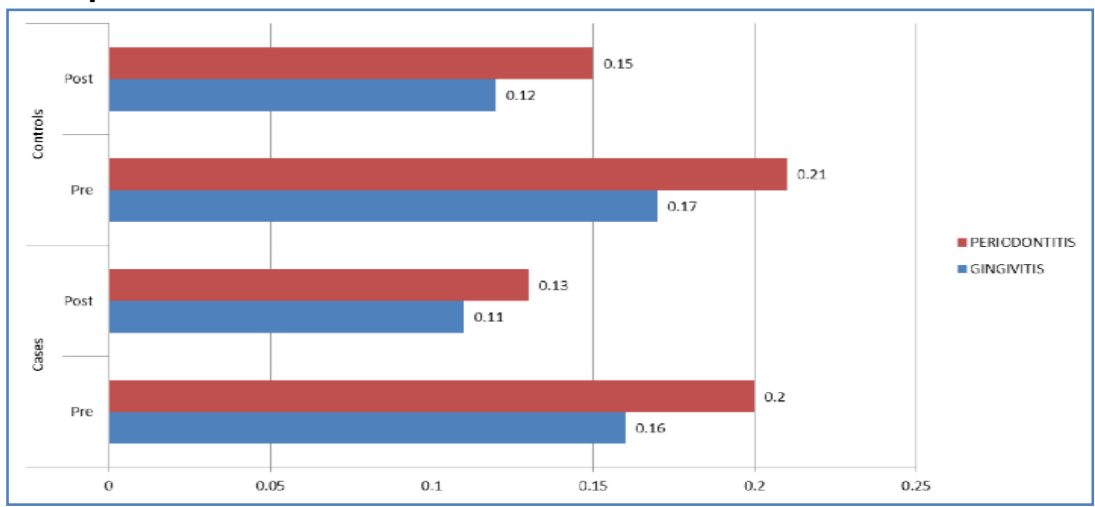

Graph 4: COMPARISON OF MPO ACTIVITY IN CASES AND CONTROLS OF GINGIVITIS AND PERIODONTITIS PATIENTS

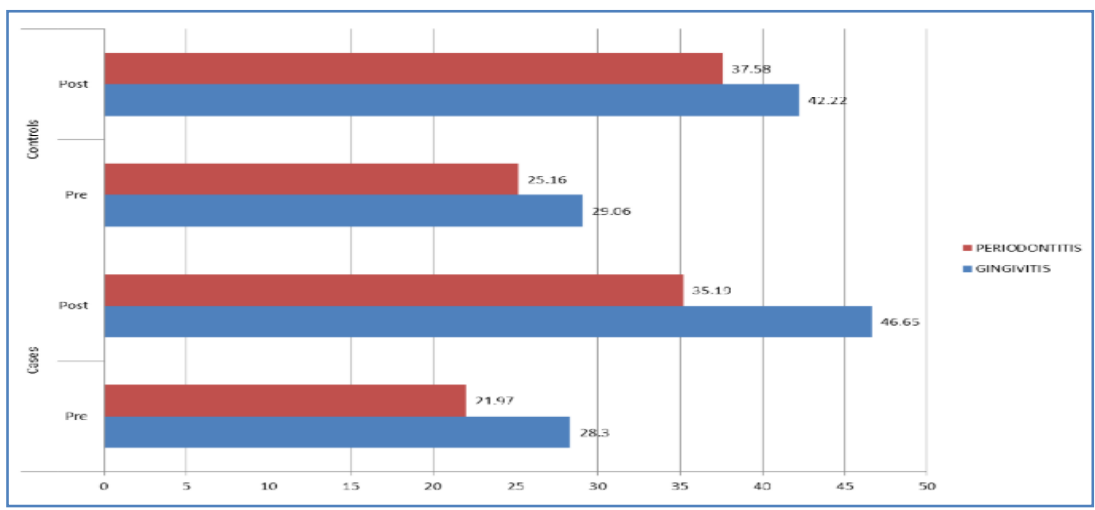

Graph 5: COMPARISON OF THIOL CONCENTRATION IN CASES AND CONTROLS OF GINGIVITIS AND PERIODONTITIS PATIENTS

\subsection{Discussion}

A greater reduction in plaque scores in the cases may be attributed to probiotic induced reduced microbial colonization. Similar results were observed by Shimauchi et al, $2008^{3}$. Krasse et al $(2006)^{4}$, Staab et al (2009) $)^{5}$ and Dhawan R et al (2013)6 corroborate the results of the present study demonstrating greater reduction of Gingival Index, in both gingivitis and periodontitis groups. Significant reduction in bleeding on probing was also noted in the Periodontitis 'Cases' group. The administration of probiotic capsules in the present study manifested anti - inflammatory effects, perceived as improvement in clinical parameters. This can be additionally attributed to the resolution of gingivitis following scaling and root planing.

It has been suggested that probiotic species effectively reduce the level of inflammation associated molecules, such as prostaglandin E2 and interferon $C$ and MMP activity in saliva (Staab et al, 2009). By modulating these factors, probiotics can lead to improvement of periodontal parameters. This may explain the greater reduction of probing depth 
and gain in clinical attachment observed in subjects supplemented with probiotics.

Significantly higher levels of MPO were observed both in gingivitis and periodontitis patients before intervention. Kowolik and Grant, $1983^{6}$, noted that MPO activity positively correlated with degree of inflammation. Periodontitis 'Cases' demonstrated greater reduction in MPO levels than controls post treatment. Anti-inflammatory effects of probiotics may have contributed to this marked improvement.

Thiol levels also significantly improved post treatment, which may be ascribed to the antioxidant capacity of probiotics. Probiotic curd administration has been shown to increase erythrocyte Superoxide dismutase, glutathione peroxidase and total antioxidant activity by Etjahed et al in 2012 . According to a recent systematic review by Mishra $\mathrm{V}$ et al in 20159 probiotics exhibit antioxidant potential. Consumption of probiotics alone or foods supplemented with probiotics may reduce oxidative damage, free radical scavenging rate, and modification in activity of crucial antioxidative enzymes in human cells.

Furthermore, no deleterious effects on general health were noted with probiotic supplementation.

The knowledge of probiotic effects on oral and general health and its administration will help the dental hygienist to effectively communicate about the dental biofilm and related aspects of plaque control. Adjunctive use of probiotics with traditional methods of brushing and flossing may help patients to acquire more efficiency in plaque control methods. This may be of relevance, specifically, in patients who lack manual dexterity.

\subsection{Conclusion:}

Probiotic supplementation had an additive effect on improvement of clinical parameters such as gingival inflammation, bleeding on probing, probing depth and gain in clinical attachment in the two treatment groups. Antioxidant activity was considerably enhanced as demonstrated by increased thiol and decreased myeloperoxidase concentrations in the gingival crevicular fluid following probiotic supplementation. Hence, probiotics may be administered as an adjunct to periodontal therapy.

It may therefore be assumed that probiotics might collectively affect the specialized bacterial ecology of the oral and intestinal tract and thus contribute immensely towards overall health.

\section{References:}

1. Kinane DF, Attström R. Advances in the pathogenesis of periodontitis consensus report of the fifth European workshop in periodontology. J Clin Perio 2005; 32: 130-131.

2. Aas JA, Paster BJ, Stokes LN, Olsen I, Dewhirst FE. Defining the normal bacterial flora in the oral cavity. J Clin Microbiol 2005; 43: 5721-5732.

3. Shimauchi $\mathrm{H}$, Mayanagi $\mathrm{G}$, Nakaya $\mathrm{S}$, Minamibuchi $\mathrm{M}$, YtoY, Yamaki K, Hirata H. Improvement of periodontal condition by probiotics with Lactobacillus salivarius WB21: a randomized, double-blind, placebocontrolled study. J Clin Periodontol 2008; 35: 897905.

4. Krasse $P$, Carlsson B, Dahl C, Paulsson A, Nilsson A, Sinkiewicz G. Decreased gum bleeding and reduced gingivitis by the probiotic Lactobacillus reuteri. Swed Dent J 2006; 30: 55-60.

5. Staab B, Eick S, Knofler G, Jentsch H. The influence of a probiotic milk drink on the development of gingivitis: A pilot study. J Clin Periodontol 2009; 36: 850-856

6. Dhawan R, Dhawan S. Role of probiotics on oral health: A randomized, double-blind, placebocontrolled study. J Interdiscip Dentistry 2013;3:71-8.

7. Kowolik MJ \& Grant M Myeloper-oxidase activity in human gingival crevicular neutrophils. Archives of Oral Biology 1983; 28: 293-295.

8. Ejtahed HS, Mohtadi-Nia J, Homayouni-Rad A, Niafar M, Asghari-Jafarabadi M, Mofid V Probiotic yogurt improves antioxidant status in type 2 diabetic patients. Nutrition. 2012; 28(5):539-43.

9. Mishra V, Shah C, Mokashe N, Chavan R, Yadav H, Prajapati J. Probiotics as potential antioxidants: a systematic review. Journal of agricultural and food chemistry. 2015 Apr 6;63(14):3615-26. 